\title{
Reduced Leishmania (L.) infantum chagasi parasitic loads in humans exposed to Lutzomyia longipalpis bites in the Amazon region of Brazil
}

\author{
MÁRCIA DALASTRA LAURENTI**1, LUIS FERNANDO CHAVES ${ }^{2,3,4}$, \\ THAÍSE YUMIE TOMOKANE ${ }^{1}$, IBRAHIM ABBASI ${ }^{5}$, ALON WARBURG $^{5}$ and \\ FERNANDO TOBIAS SILVEIRA ${ }^{6,7}$ \\ ${ }^{1}$ Laboratory of Pathology of Infectious Diseases, Department of Pathology, Medical School, University of São Paulo, \\ São Paulo (SP), Brazil \\ ${ }^{2}$ Centro de Investigación en Enfermedades Tropicales, Universidad de Costa Rica, San Pedro de Montes de Oca, \\ Costa Rica \\ ${ }^{3}$ Programa de Investigación en Enfermedades Tropicales (PIET), Escuela de Medicina Veterinaria, Universidad Nacional, \\ Heredia, Costa Rica \\ ${ }^{4}$ Institute of Tropical Medicine (NEKKEN), Nagasaki University, Nagasaki, Japan \\ ${ }^{5}$ The Department of Microbiology and Molecular Genetics, The Kuvin Center for the study of Infectious and Tropical \\ Diseases, The Institute of Medical Research Israel-Canada, The Hebrew University of Ferusalem, Ferusalem, Israel \\ ${ }^{6}$ Instituto Evandro Chagas, Ministry of Health, Ananindeua (PA), Brazil \\ ${ }^{7}$ Nucleous of Tropical Medicine, Federal University of Pará, Belém (PA), Brazil
}

(Received 23 September 2016; revised 3 February 2017; accepted 6 February 2017)

S U M MARY

We performed a cross-sectional survey in humans to evaluate Lutzomyia longipalpis, i.e. sand fly vector, bite exposure association with Leishmania (Leishmania) infantum chagasi infection in Bujarú municipality, Northern Brazil, an endemic area for visceral leishmaniasis. In recruited individuals, which were stratified by sex and age, we measured $L$. (L.) $i$. chagasi parasitic loads with quantitative polymerase chain reaction (qPCR), exposure to sand fly bites with an anti-saliva immunoglobulin $\mathrm{G}$ enzyme-linked immunosorbent assay and performed immunological diagnostic tests, in order to evaluate the association between exposure to sand fly bites, and infection. The prevalence increased from $11 \%$ when using immunological diagnostic tests to $28 \%$ when using qPCR, being around that value for all age classes, but children below 5 years $(40 \%)$ and people over 60 years $(15 \%)$. The association between PCR-based $L$. (L.) i.chagasi prevalence and saliva exposure was convex, reflecting the fact that at both high and low saliva exposure the PCR-based $L$. (L.) $i$. chagasi prevalence decreases. This scenario indicates that low sand fly exposure is likely associated with low parasite transmission, while high anti-saliva prevalence, i.e. a large sand fly bite exposure could be associated with anti-Leishmania protective immune mechanisms driven by vector saliva and/or increased parasite exposure.

Key words: visceral Leishmaniasis, diagnosis, pathogenesis, protozoan infections, sand flies.

\section{INTRODUCTION}

Visceral leishmaniasis (VL) is a systemic protozoan infection caused by intracellular Leishmania parasites. An estimated $500000 \mathrm{VL}$ cases occur annually, over $90 \%$ of which are concentrated in the Indian subcontinent, East Africa and Brazil (Desjeux, 2001, 2004). Three species of Leishmania belonging to the L. donovani complex, cause VL throughout most of its distribution; Leishmania (Leishmania) donovani and L. (L.) infantum in the Old World and $L$. (L.) infantum chagasi in the New World (Lainson and Shaw, 2005). In the New World, the most important vector is the sand fly Lutzomyia

* Corresponding author: Laboratório de Patologia de Moléstias Infecciosas, Faculdade de Medicina da Universidade de São Paulo, Av. Dr. Arnaldo, 455 - 1o andar - sala 1209, CEP: 01246-903, São Paulo (SP), Brazil. E-mail: mdlauren@usp.br longipalpis (Diptera: Psychodidae) (Salomón et al. 2015).

VL was confined to rural areas in North-Eastern Brazil until the early 1980s. However, over the past 30 years increasing levels of transmission have been recorded in peri-urban and even fully urbanized areas, including large cities in geographically distant parts of the country. This trend is most likely facilitated by large-scale migration of extremely poor populations to the cities where unplanned shanty-towns accommodate people and domestic animals under crowded and unhygienic conditions (Maia-Elkhoury et al. 2008; Harhay et al. 2011). Crucial to the spread of disease is the fact that Lu. longipalpis, the main sand fly vector of $\mathrm{VL}$ is highly adaptable to modified peri-urban and urban settings (Costa, 2008; Amora et al. 2010; Saraiva et al. 2011).

Infections with $L$. (L.) $i$. chagasi in humans can result in variable conditions ranging from

Parasitology Open (2017), Vol. 3, e2; page 1 of 10. (C) Cambridge University Press 2017. This is an Open Access article, distributed under the terms of the Creative Commons Attribution licence (http://creativecommons.org/licenses/by/4.0/), which permits unrestricted re-use, distribution, and reproduction in any medium, provided the original work is properly cited. 
asymptomatic cases through subclinical infections to classic VL. The prevalence of asymptomatic carriers in endemic areas is highly variable. The use of more than one technique increases the number of asymptomatic cases detected and allows their classification into sub-groups (Michel et al. 2011). In Brazil, the Leishmanin skin test (LST), specific to the type of cell-mediated response (DTH, type IV hypersensitivity) has been used to detect $L$. (L.) $i$. chagasi infection in endemic areas; as well as enzymelinked immunosorbent assay (ELISA) and indirect fluorescent antibody test (IFAT) are also commonly for detecting specific anti-Leishmania antibodies. In more recent studies, Leishmania-specific polymerase chain reaction (PCR)-based diagnostics are increasingly being used (Garcia et al. 2004).

Levels of exposure to sand fly bites can be estimated by monitoring the seroconversion to sand fly saliva. Experimental studies in animal models have shown that repeated exposure to sand fly bites induces sand fly species-specific anti-saliva antibodies detectable by ELISA and Western blot (Volf and Rohoušová, 2001). Similarly, the presence of antibodies to sand fly has been documented in humans naturally exposed to sand flies bites (Kamhawi et al. 2000); and a positive association between anti-saliva immunoglobulin $\mathrm{G}$ ( $\mathrm{IgG})$ response and cell-mediated response to Leishmania was also noted (Barral et al. 2000; Gomes et al. 2002).

Here, we present results from a cross-sectional epidemiological survey in the human population from an endemic rural VL from Brazilian Amazon region. In the recruited individuals, we measured $L$. (L.) $i$. chagasi parasitic loads, exposure to sand fly bites (measuring anti-saliva IgG antibodies) and performed classical diagnostic tests, in order to evaluate the association between exposure to sand fly bites, $L$. (L.) $i$. chagasi infection and clinicalimmunological profiles, in a population stratified by sex and age. So, this study helps to understand VL transmission ecology, especially when linked with epidemiological data on the infection patterns of a human population.

\section{MATERIALS AND METHODS}

\section{Study area}

This study was carried out in Bujarú municipality, Pará State, Brazil. The climate is typically tropicalequatorial, with an average temperature of $28^{\circ} \mathrm{C}$ and high humidity. The annual rainfall in the region is around $2500 \mathrm{~mm}$, the rainy season spanning from January to June. Following extensive destruction of the primary forest, the area now consists mainly of plantations, with fragmented patches of developing secondary forest. Our field survey was conducted from March to May (2012). This period coincides with the highest abundance season
(January-May) for Lu. longipalpis, the vector of $L$. (L.) $i$. chagasi in this region, also the most abundant sand fly species in the area (Lainson and Rangel, 2005). In March-May of 2011, we estimated a $13 \%$ prevalence of $L$. (L.) $i$. chagasi infections in humans (F.T. Silveira, personal communication, 2012).

\section{Cross-sectional survey}

Individual enrolment in the survey was comprehensive among the different villages, and representative of age groups and gender composition of Bujarú. We enrolled a total of 414 individuals belonging to 107 families. Venous blood $(5 \mathrm{~mL})$ was drawn by a qualified clinician from all individuals. Four drops were blotted on filter paper, which was used for PCR tests, and the rest of the blood was kept in a tube for separation of sera for anti-L. (L.) $i$. chagasi IFAT and anti-Lu. longipalpis saliva ELISA. LSTs were also performed and monitored after $72 \mathrm{~h}$. From the original 414 samples determining levels of anti-saliva antibodies, the test could not be performed on 15 samples since there was not enough serum to test. For 69 individuals the age was not recorded, due to lack of consent. Thus, most of the subsequent analyses will be based on the 332 individuals for which there were no missing data about age or anti-saliva antibodies.

\section{Ethical clearance}

Informed consent was sought and obtained from all persons volunteering for the study - or parents of minors participating. This study was approved by the Ethics Committee for human research of the São Paulo University Medical School under protocol number \#184/12.

\section{Diagnostic tests}

Clinical exam. All individuals presenting any positive immunological reaction, either LST or IFAT, were clinically examined in order to identify any signs and/or symptoms of VL. The subclinical oligo-symptomatic cases were followed-up during 6 months to confirm their spontaneous clinical resolution. The clinical-immunological profile of infection was based on semi-quantitative analysis with scores varying from + to ++++ , as described by Crescente et al. (2009).

Leishmanin Skin Test. The LST was performed using cultured stationary phase $L$. (L.) i chagasi promastigotes (MCAO/BR/2003/M22697/Barcarena, PA, Brazil). Promastigotes were fixed using 0.01\% Merthiolate $^{\circledR}$ solution. LSTs were performed by intradermal injection of $0 \cdot 1 \mathrm{~mL}$ antigen $\left(10^{7}\right.$ parasites $\left.\mathrm{mL}^{-1}\right)$ and reactivity was scored $72 \mathrm{~h}$ thereafter 
(Crescente et al. 2009; Silveira et al. 2010). An induration of $\geq 16 \mathrm{~mm}$ diameter at the site of injection was assigned a score of ++++ , strongly positive $(13-15 \mathrm{~mm})+++$, moderately positive $(9-12 \mathrm{~mm})$ ++ , and weakly positive $(5-8 \mathrm{~mm})+$. This test has been proved as highly specific and sensitive, 100\%, for immune responses against $L$. (L.) $i$. chagasi associated with CD4+ Th1 cells (Silveira et al. 2010).

Indirect fuorescent antibody test. Small sections of L. (L.) infantum chagasi-infected hamster spleen or liver were used to do imprints in multi-well IFAT slides. After dry the imprints were fixed in acetone for $15 \mathrm{~min}$; and then they were washed in phosphate buffered saline (PBS) followed by incubation at $37^{\circ} \mathrm{C}$ for $30 \mathrm{~min}$ with test sera (in serial dilution starting at $1 / 40$ in PBS). After PBS washing, the slides were incubated with anti-human IgG-FITC (Sigma Aldrich, USA) diluted 1/100 (Lima et al. 2003). Followed by another washing, the slides were mounted with glycerin and the reaction was assessed using fluorescence microscopy. Based on the intensity of fluorescence serological titres $(\mathrm{IgG})$ with $80-160$ and 320-640 received + and ++ and those with 1280-2560 and 5120-10 240, +++ and ++++ , respectively. This test has a reported sensitivity of $96 \%$ and specificity of $100 \%$, when used against a gold standard of parasite observation (Lima et al. 2003).

\section{Sand fly bite exposure ELISA}

To measure sand fly bite exposure, the level of antiLu. longipalpis saliva antibodies was evaluated. Lutzomyia longipalpis is the dominant species, and the main vector responsible for $L$. (L.) $i$. chagasi transmission, in peri-domestic environments in the study area. Sand fly bite exposure was evaluated by ELISA according to the method described by Rohousova et al. (2005), with a few changes. Briefly, microtitre plate wells (High binding, Nunc) were coated with Lu. longipalpis salivary gland lysate $(60 \mathrm{ng}$ of protein in $20 \mathrm{mM}$ carbonatebicarbonate buffer, $\mathrm{pH} 9 \cdot 6$ ) overnight at $4{ }^{\circ} \mathrm{C}$. The wells were then washed in PBS with $0.05 \%$ Tween-20 (PBS-T) and incubated with 10\% skimmed milk powder in PBS for $60 \mathrm{~min}$ at $37^{\circ} \mathrm{C}$ to block free binding sites. Human sera were diluted 1:50 in PBS-T, and incubated in duplicates $90 \mathrm{~min}$ at $37^{\circ} \mathrm{C}$. Wells were incubated with peroxidase-conjugated anti-human $\mathrm{IgG}$ secondary antibody (Sigma Aldrich, USA) at a 1:2000 dilution for $45 \mathrm{~min}$ at $37^{\circ} \mathrm{C}$ and revealed using tetramethilbenzidine (TMB) (Becton Dickinson Bioscience, USA). The $2 \mathrm{~N}$ sulphuric acid solution was used to stop the reaction and the absorbance was measured using an ELISA reader at $450 \mathrm{~nm}$. We also performed this test to 47 individuals from São Paulo, Brazil, a non-endemic area for VL, which were used as controls. In this external population, 30 individuals have never been to areas with sand flies, and 17 have been exposed to sand flies' bites during their life time. Among the later, 10 samples were from volunteers exposed to Lu. longipalpis bites for feeding laboratory reared Lu. longipalpis females, at least once a month for more than 1 year, while all the other seven positive controls recalled being bitten by sand flies.

\section{Real-time PCR}

Quantitative PCR (qPCR) was based on the minicircles kinetoplast DNA (Nicolas et al. 2002). DNA was extracted using a Promega DNA extraction kit, from the blood samples eluted from the filter papers, Whatman 3MM (GE Healthcare Life Sciences, USA). An RT-PCR for k-DNA was performed for each sample using the Chromo 4 (Bio-Rad, CA, USA) with the Dynamo HS, SYBR green qPCR kit (Finnzymes, Thermo Fisher Scientific, USA). Primers JW11 (5'-CCTATTTTACACCAACCCC CAGT-3') and JW12 (5'-GGGTAGG GGCGT TCTGCGAAA-3') were used to amplify a $120 \mathrm{bp}$ fragment of the kinetoplast minicircle as described elsewhere (Abbasi et al. 2013). The specificity of the PCR is $92 \%$, while its sensitivity is differential according to the parasitic loads, $59 \%$ for loads of 1-10 parasites $\mu \mathrm{L}^{-1}, 85 \%$ for loads between 11 and 100 parasites $\mu \mathrm{L}^{-1}$, 96\% for loads $101-1000$ parasites $\mu \mathrm{L}^{-1}$ and $100 \%$ for loads above 1000 parasites $\mu \mathrm{L}^{-1}$, where loads over 100 parasites $\mu \mathrm{L}^{-1}$ correspond to loads of patients where direct parasite observation is reliable (Abbasi et al. 2013).

\section{Statistical analysis}

Seropositivity for anti-Lu. longipalpis saliva antibodies (Sandfly saliva exposure). To assign individuals as seropositive or seronegative we employed a mixture model (Calzada et al. 2015). This model is used to estimate parameters for two distributions, i.e. one for seropositive and one for seronegative individuals, which are assumed to get mixed and be represented by the overall distribution of optical densities measured for an ELISA test (Calzada et al. 2015). A threshold for seropositivity can then be obtained by estimating the 99.99 percentile of the distribution for seronegative individuals, which is computed by adding three times the S.D. to the mean of the distribution with the lowest mean, which is the one assumed for seronegative individuals (Calzada et al. 2015). The thresholds found with the mixture model were then further evaluated with the positive and negative controls from São Paulo, which were not considered when fitting the model.

PCR-based prevalence as a function of age and sand fly saliva exposure. Data for individuals with complete information $(n=332)$ were summarized by 
groups that correspond to deciles in the age distribution of the studied population. The resulting data were then analysed using a linear regression (Faraway, 2004), where the PCR prevalence was a function of the median age in each age decile and the prevalence of exposure to sand fly saliva.

Leishmania parasitic loads as a function of age, sex and sand fly saliva exposure. Data for the parasitic loads from each individual were analysed as function of their age, sex and exposure to sand fly saliva. For saliva exposure we considered a model that used a categorical covariate: saliva exposed/unexposed and compared it with a model that used the raw optical density data from the saliva exposure ELISA test. We choose the best model based on the minimization of the Akaike information criterion (AIC) (Faraway, 2004). The AIC is a metric based on the trade-off between goodness of fit and parameter number in a statistical model (Faraway, 2004). Since parasitic loads are counts, and data came from individuals who belonged to a set of families, thus making the data not fully independent (Chaves, 2010), we used a Poisson generalized linear mixed model, i.e. a Poisson GLMM (Finch et al. 2014) to account both for the count nature of the data and the lack of independence, using families as a random factor in the analysis. The Poisson GLMM was fitted using the Laplace approximation for maximum likelihood (Bolker et al. 2009).

Association between leishmaniasis clinical condition, leishmaniasis diagnostic tests, sand fly saliva exposure and sex. Data on seropositivity to sand fly saliva,
IFAT, LST, parasite presence (PCR positivity) and sex, were studied for their association with different leishmaniasis clinical conditions. Given the categorical nature of the data (individuals being positive or negative, males or females), we performed a multiple correspondence analysis to depict the association between the different tests. In this multivariate statistical method, a data matrix is created where each row corresponds to data from a given individual. This matrix is then subjected to a singular value decomposition, which allows the representation of the association into a plane by projecting the original data into the two vectors with the largest singular values (Venables and Ripley, 2002). In this analysis, centroids for the levels of the different categorical variables can be plotted into the plane, and their proximity is a measurement of their association, which is strongest as levels from a category are close together but far apart from the origin of the plane, i.e. when the abscise and ordinate axes equal 0 , the point where randomly associated variables are expected to appear (Venables and Ripley, 2002).

Finally, all statistical analyses and figures were made with the statistical software $\mathrm{R}$ version 3.2.2.

\section{RESULTS}

\section{Clinical-immunological profile of L. (L.) i. chagasi infection}

Table 1 shows the distribution of $L$. (L.) $i$. chagas $i$ infected and non-infected individuals according to age and sex based on clinical evaluations, LST and IFAT. From 414 individuals, 46 were positive for

Table 1. Distribution of clinical-immunological profiles of human Leishmania (Leishmania) infantum chagasi infection according to age, sex and parasitological diagnosis by PCR in Bujarú municipality, state of Pará, Brazil

\begin{tabular}{|c|c|c|c|c|c|c|c|}
\hline & \multirow[b]{2}{*}{ Total $n(\%)$} & \multirow[b]{2}{*}{ Healthy group $n(\%)$} & \multicolumn{5}{|c|}{ Clinical-immunological profile of infection $[n(\%)]$} \\
\hline & & & SI & AI & SOI & SRI & III \\
\hline \multicolumn{8}{|l|}{ Age (years) } \\
\hline Non-recorded & $69(16)$ & $62(15 \cdot 0)$ & $0(0)$ & $2(0 \cdot 5)$ & $0(0)$ & $1(0 \cdot 2)$ & $4(1 \cdot 0)$ \\
\hline $0-10$ & $112(27)$ & $106(25 \cdot 6)$ & $0(0)$ & $3(0 \cdot 7)$ & $0(0)$ & $1(0 \cdot 2)$ & $2(0 \cdot 5)$ \\
\hline $11-20$ & $89(22)$ & $80(19 \cdot 3)$ & $0(0)$ & $7(1 \cdot 7)$ & $0(0)$ & $0(0)$ & $2(0 \cdot 5)$ \\
\hline$\geq 21$ & $144(35)$ & $120(29 \cdot 0)$ & $0(0)$ & $19(4 \cdot 6)$ & $1(0 \cdot 2)$ & $3(0 \cdot 7)$ & $1(0 \cdot 2)$ \\
\hline \multicolumn{8}{|l|}{ Sex } \\
\hline Male & $194(47)$ & $167(40 \cdot 3)$ & $0(0)$ & $17(4 \cdot 1)$ & $0(0)$ & $3(0 \cdot 7)$ & $7(1 \cdot 7)$ \\
\hline Female & $220(53)$ & $201(48 \cdot 6)$ & $0(0)$ & $14(3 \cdot 4)$ & $1(0 \cdot 2)$ & $2(0 \cdot 5)$ & $2(0 \cdot 5)$ \\
\hline \multicolumn{8}{|l|}{ PCR } \\
\hline Positive & $114(27 \cdot 6)$ & $100(24 \cdot 2)$ & $0(0)$ & $12(2 \cdot 9)$ & $0(0)$ & $0(0)$ & $2(0 \cdot 5)$ \\
\hline Negative & $300(72 \cdot 4)$ & $268(64 \cdot 7)$ & $0(0)$ & $19(4 \cdot 6)$ & $1(0 \cdot 2)$ & $5(1 \cdot 2)$ & $7(1 \cdot 7)$ \\
\hline
\end{tabular}

The clinical-immunological profile was based on semi-quantitative LST and IFAT considering an indirection area $\geq 5 \mathrm{~mm}$ and 1/80 titre, respectively, as positive cut-off for immunological tests according to Crescente et al. (2009), where: AI, asymptomatic infection (LST $+/++++$ and IFAT-); SI, symptomatic infection and SOI, subclinical oligo-symptomatic infection (LST - and IFAT+++/++++); SRI, subclinical resistant infection (LST+/++++ and IFAT+/++); and III, initial indeterminate infection $(\mathrm{LST}-$ and IFAT $+/++)$. PCR, polymerase chain reaction used to search Leishmania DNA in peripheral blood. 
at least one immunological test and 368 were negative for both of them, reflecting a prevalence of $11 \%$. Considering an induration of $>5 \mathrm{~mm}$ diameter. for LST and 1/80 titre for IFAT, as positive cut-off criteria for immunological tests, we observed a prevalence of $7 \cdot 5 \%$ for asymptomatic infections (AIs), $0 \cdot 2 \%$ for subclinical oligo-symptomatic infections (SOIs), $1 \cdot 1 \%$ for subclinical resistant infections (SRIs) and $2 \cdot 2 \%$ for initial indeterminate infection (III). During the survey conducted from March to May 2012 no symptomatic AVL cases were recorded. PCR results indicated that $27 \cdot 6 \%$ of the volunteers were infected with $L$. (L.) $i$. chagasi. In addition, many individuals from endemic areas negative for clinical signs, LST and IFAT (healthy group) were positive by PCR (100/368).

\section{Distribution of anti-Lu. longipalpis saliva antibody titres}

To assign individuals as seropositive or seronegative for anti-saliva antibodies we used data from 399 individuals. Figure 1 shows the distribution of the optical densities employed for this analysis, of which 350 were seronegative and 49 seropositive, roughly indicating a $25 \%$ seroprevalence of

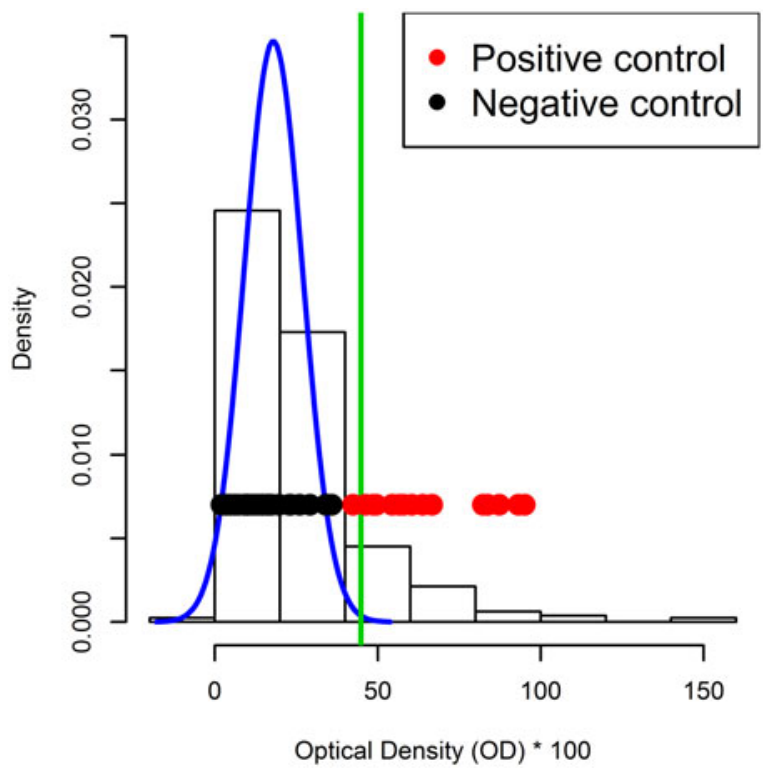

Fig. 1. Histogram for the optical density (OD) distribution of the anti-Lu. longipalpis saliva antibodies. The blue line indicates the fitted distribution for negative individuals, estimated using a mixture model. The vertical green line indicates the 95 percentile of the negative distribution, which is used as a threshold, i.e. an OD above $44 \cdot 83$, to assign individuals as seropositive for saliva antibodies when their OD is above the threshold. Black dots are the ODs of negative control individuals from São Paulo, Brazil never exposed to sand flies, while red dots are the ODs positive control individuals from São Paulo, Brazil exposed to sand fly bites. The y axis is the probability density for the antibody ODs in the $x$-axis. anti-saliva antibodies. Regarding the external controls, we found that all unexposed individuals $(n=30)$ were classified as unexposed to sand fly saliva, i.e. a specificity of $100 \%$, while one sand fly saliva exposed individual $(n=17)$ was classified as unexposed, the rest of the individuals being properly classified as exposed to sand fly saliva, i.e. a sensitivity of $94 \cdot 1 \%$. The false negative was from a positive control involved in blood feeding $L u$. longipalpis sand fly colonies.

L. (L.) i. chagasi $D N A$ and anti-Lu. longipalpis saliva IgG positivity in different age groups

Figure 2 shows the L. (L.) infatum chagasi prevalence as a function of age. In general, it can be seen that only at extreme young (below 5 years) and old (above 60 years) ages the prevalence is different from all other age groups which fluctuates around $28 \%$. For extreme young ages, the prevalence is higher $(\sim 40 \%)$ and for old ages (above 60$)$ is lower at around $15 \%$. A similar pattern is shown in Fig. 3 for the prevalence of sand fly bite exposure, yet values are smaller than those recorded for parasite prevalence for the same age groups.

Association between L. (L.) i. chagasi $D N A$ and anti-Lu. longipalpis saliva IgG

Figure 4 shows the association between the PCRbased $L$. (L.) $i$. chagasi prevalence and exposure to sand fly bites (anti-saliva seropositivity). The association is convex, reflecting the fact that at both high and low anti-saliva seroprevalence the PCR-based L. (L.) i. chagasi prevalence decreases. The nonlinear

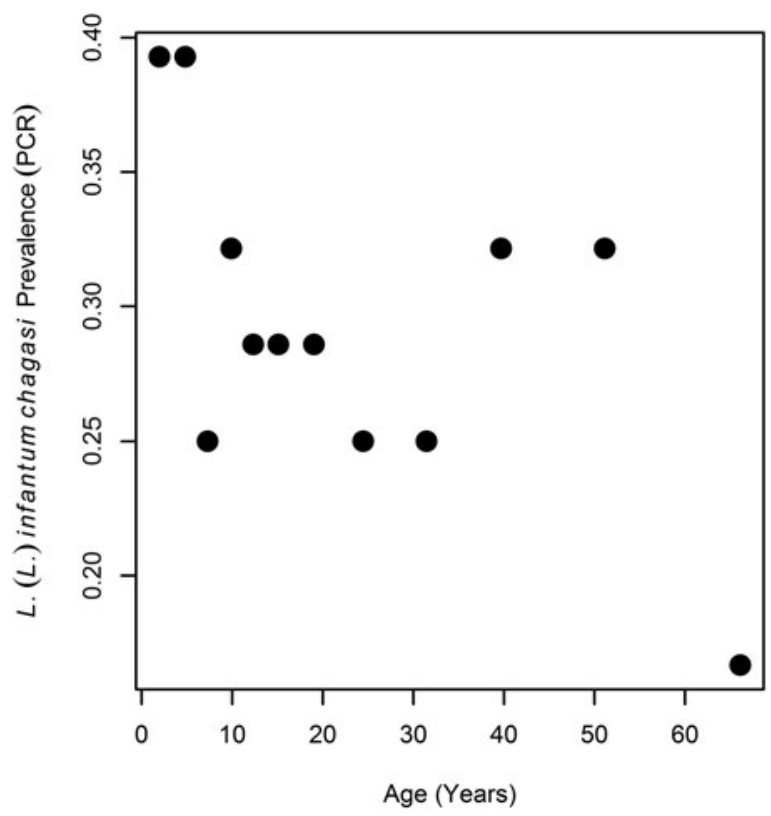

Fig. 2. Leishmania (Leishmania) infantum chagasi PCR prevalence as function of age. 


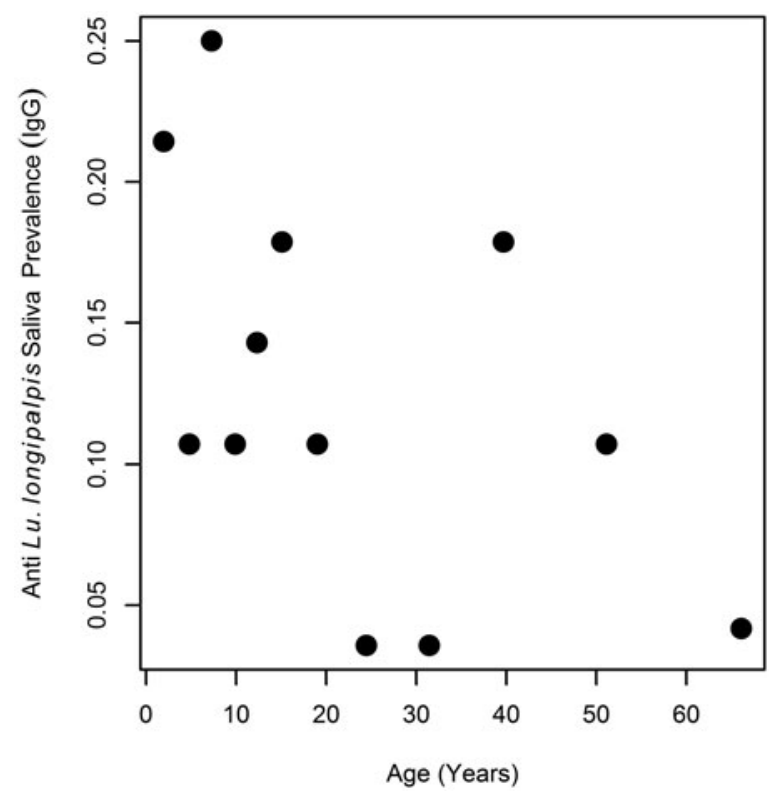

Fig. 3. Anti-Lutzomyia longipalpis saliva IgG prevalence as function of age.

relationship of $L$. (L.) $i$. chagasi parasite prevalence with age (Fig. 2), where around 10 years of age parasite prevalence reduction with age seemed to change its rate in a disruptive fashion, can be modelled using a hockey stick function, HSF (Faraway, 2004). Briefly, with a HSF one parameter is estimated for points below a tipping point (here 10 years), and one for points above the tipping point (i.e. 10 years), the two curves joining at the tipping point (i.e. 10 years). Technical details about the construction of HSF are explained in detail by Faraway (2004) and elsewhere (Chaves et al. 2012). The

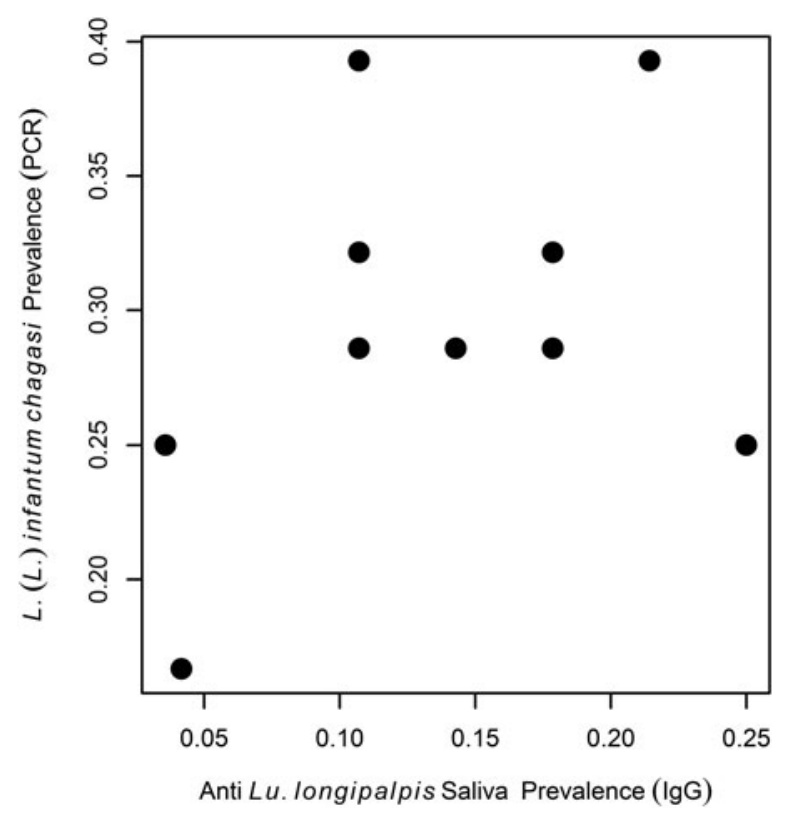

Fig. 4. Leishmania (Leishmania) infantum chagasi PCR prevalence as function of anti-Lutzomyia longipalpis saliva IgG prevalence.
Table 2. Parameter estimates for a linear regression depicting the association between Leishmania (Leishmania) infantum chagasi parasite prevalence, the exposure to sand fly saliva (saliva prevalence) and age

\begin{tabular}{lrlrc}
\hline \hline Parameters & Estimate & S.E. & $t$ value & $\operatorname{Pr}(>|t|)$ \\
\hline $\begin{array}{l}\text { Intercept } \\
\begin{array}{l}\text { Saliva } \\
\text { prevalence }\end{array}\end{array}$ & 0.4313 & 0.0410 & 10.509 & $>0.00001^{*}$ \\
$\begin{array}{l}\text { (Saliva } \\
\text { prevalence) }\end{array}$ & -0.1320 & 0.0340 & -3.885 & $0.00601^{*}$ \\
$\begin{array}{l}\text { LHS (Age) } \\
\text { RHS (Age) }\end{array}$ & -0.0147 & 0.0058 & -2.510 & $0.04042^{*}$ \\
Error variance & 0.0335 & 0.0044 & -3.673 & $0.00793^{*}$ \\
\hline \hline
\end{tabular}

The ${ }^{2}$ indicates the square of a covariate. LHS and RHS, indicate the Left Hockey Stick (which is equal to age when it is below 10 years of age, 0 otherwise) and Right Hockey Stick (which is equal to age when it is above 10 years of age, 0 otherwise) function of age.

*Statistically significant $(P<0 \cdot 05)$.

relationship between parasite prevalence and saliva exposure was convex (Fig. 4), which suggested the use of a 2 nd degree polynomial to describe the association. Table 2 shows the results of the linear regression of parasite prevalence as function of age and sand fly saliva exposure. This model explained $82 \%$ of the variability in the data $\left(R^{2}=0 \cdot 82 ; F_{4,7}=\right.$ $8 \cdot 074, P<0 \cdot 00925)$. In the model, parameter estimates suggest a slight change in the parasite prevalence rate around 10 years of age, the small change suggest that age patterns presented in Figs 2 and 3 is likely mainly driven by a differential exposure to sand fly bites. The negative parameter for the squared term in the second degree polynomial, suggests that parasite prevalence increases with the exposure to sand fly bites/saliva until reaching a maximum, above which parasite prevalence decreases with sand fly exposure.

L. (L.) i. chagasi blood parasitemias as a function of anti-Lu. longipalpis saliva $\operatorname{IgG}$ optical density

Figure 5 shows L. (L.) i. chagasi blood parasitaemias as a function of the anti-Lu. longipapis saliva $\mathrm{Ab}$ titres (ELISA). It can be seen that, in general, parasitaemias decreased with higher titres of anti-saliva Abs, and that positive individuals for parasitic infections were more likely to be non-reactive to sand fly saliva. The Poisson GLMM analysis (Table 3 ) indicates that males were likely to have $3 \cdot 4$ times more parasites than females, that mean parasitaemias decreased by about $1 \%$ with each year of age and that parasitaemias of individuals exposed to sand fly saliva were reduced by $75 \%$ when compared with unexposed individuals. This model minimized the AIC (AIC = 5263) when compared with a model that used optical density from the saliva ELISA as covariate $(\mathrm{AIC}=5303)$. 


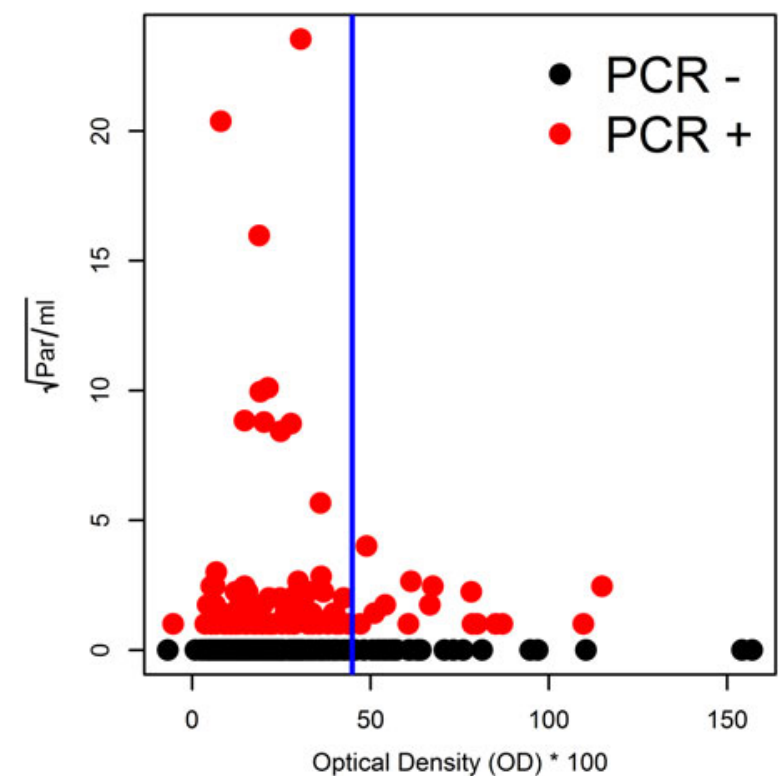

Fig. 5. Leishmania (Leishmania) infantum chagasi parasite loads in individuals from endemic area as function of the optical density of anti Lutzomyia longipalpis saliva IgG. The vertical blue line is the threshold for the exposure to sand fly saliva antigens, dots to the left of this line being negative, and positive to the right. Dot colour indicates whether the PCR was positive or negative (see inset legend). In the $Y$-axis parasitic loads were square root transformed to ease visualization.

Multiple correspondence analysis considering clinicalimmunological profile and diagnostic tests for L. (L.) i. chagasi infection

Figure 6 shows the results of a multiple correspondence based on the 292 individuals for which information on all tests was available. The figure shows that in general the lack of clinical signs, sex, seropositivity for anti-saliva antibodies were associated in a fashion not different from what is expected by random. In contrast, individuals who tested positive in the DTH/LST tests were likely to show AI clinical-immunological profile, while individuals who test positive for IFAT were associated with III and SOI clinical-immunological profiles. Finally,

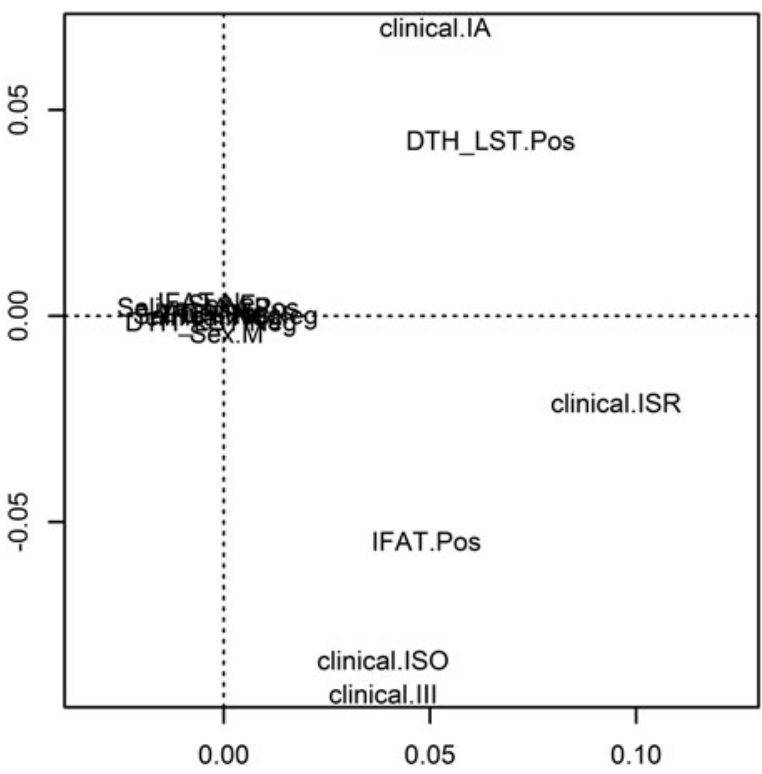

Fig. 6. Multiple correspondence analyses considering the clinical-immunological profiles of Leishmania (Leishmania) infantum chagasi infection, seropositivity for Lutzomyia longipalpis saliva, seropositivity for $L$. (L.) i. chagasi (IFAT), Leishmanin skin test (LST), L. (L.) i. chagasi PCR positivity results and gender (i.e. patient sex). The cumulative variance explained by the two dimensions projected is $22 \%$. For the clinicalimmunological profiles: AI stands for asymptomatic infection, RSI stands for resistant subclinical infection, OSI for oligo-symptomatic subclinical infection and III for initial indeterminate infection. LST.Pos and IFAT. Pos stand, respectively, for positive DTH/LST and IFAT tests. The cumulative variance explained by the two dimensions projected is $22 \%$.

individuals with SRI clinical-immunological profile were likely to be positive by either DTH/LST or the IFAT test.

\section{DISCUSSION}

Our cross-sectional epidemiological survey in humans from an endemic rural VL area in Brazilian Amazon region showed the following distribution of $L$. (L.) $i$. chagasi infections when clinical signs

Table 3. Parameter estimates for a Poisson generalized linear mixed model for the Leishmania (Leishmania) infantum chagasi parasitic loads as function of age, sex and exposure to sand fly saliva

\begin{tabular}{lllllr}
\hline \hline Parameter estimates & Rate change & Estimate & S.E. & $z$ value & $\operatorname{Pr}(>|z|)$ \\
\hline Fixed factors & & & & & \\
$\quad$ Females-saliva negative & - & $-1 \cdot 454$ & $0 \cdot 318$ & $-4 \cdot 57$ & $4 \cdot 93 \times 10^{-6}$ \\
$\quad$ Males & $3 \cdot 371$ & $1 \cdot 215$ & $0 \cdot 0603$ & $20 \cdot 16$ & $<2 \times 10^{-2 *}$ \\
$\quad$ Age & 0.989 & $-0 \cdot 0107$ & $0 \cdot 00188$ & $-5 \cdot 67$ & $1 \cdot 46 \times 10^{-8 *}$ \\
$\quad$ Saliva positive & $0 \cdot 253$ & $-1 \cdot 373$ & $0 \cdot 155$ & $-8 \cdot 86$ & $<2 \times 10^{-16 *}$ \\
Random factor & & $5 \cdot 292$ & & & \\
$\quad$ Family variance & & & & \\
\hline \hline
\end{tabular}

In this model the family $(n=95)$ to which individuals $(n=332)$ belonged was considered as a random factor.

* Statistically significant $(P<0 \cdot 005)$. 
were considered, LST and IFAT showed a prevalence of $7 \cdot 5 \%$ for $\mathrm{AI}, 0 \cdot 2 \%$ for $\mathrm{SOI}, 1 \cdot 1 \%$ for SRI and $2 \cdot 2 \%$ for III, similar to what has been previously observed in the study area (Silveira et al. 2009, 2010).

In addition, from 414 individuals evaluated, 46 showed at least one positive immunological test reflecting in a total prevalence of $11 \%$. However, according to PCR positivity, $L$. (L.) $i$. chagasi infection prevalence was $27 \cdot 6 \%$, some $16 \%$ higher, highlighting the frequent occurrence of totally AIs, so these results confirmed the high sensitivity of PCR for diagnosis of leishmaniasis. Our results showed that PCR is a good test for diagnosis of infection, since it detects cases without any apparent clinical signs and immune reaction (Solano-Gallego et al. 2011).

In relation to $L$. (L.) $i$. chagasi prevalence as function of age, it can be seen that only for extreme young (below 5 years) and old (above 60 years) age groups the prevalence is different from all other age groups, which fluctuate around $28 \%$. For extreme young ages, the prevalence is higher near to $40 \%$ and for old ages (above 60 ) is lower at around $15 \%$. This scenario indicates endemic transmission, with higher prevalence in young children and low prevalence in old people, but the nearly constant prevalence in age groups corresponding to the economically active population, which in the area has a large proportion of 'economic' migrants, i.e. people who moved for jobs and now reside into the area permanently (Prothero, 1965; Wijeyaratne et al. 1994; Confalonieri et al. 2014).

These results suggest that migrants into the area are highly exposed to $L$. (L.) $i$. chagasi infections and might account for the flat prevalence observed for these age groups (Anderson and May, 1991), as is commonly observed when a pathogen newly invades an area, or when a new susceptible population arrives to an endemic area Kaneko et al. 1998, 2014). This possibility is re-enforced by the similar pattern observed for the prevalence of sand fly bite exposure, which indicates endemic transmission, showing higher prevalence in young children and low prevalence in old adults (Anderson and May, 1991; Calzada et al. 2015), with a flat prevalence for age groups corresponding to the economically active population, which has a high proportion of migrants in our study area (Confalonieri et al. 2014).

The convex relationship between $L$. (L.) $i$. chagasi prevalence and sand fly saliva exposure likely indicates the following scenarios: (i) at low saliva prevalence, little sand fly bite and parasite exposure, therefore there is no transmission and no parasites are detected by PCR; and (ii) at high saliva prevalence, a lot of exposure, likely resulting on immune protection from both the saliva and exposure to the leishmanial parasites, confirming what was already suggested by the data of $L$. (L.) $i$. chagasi PCR positivity as function of $L u$. longipalpis saliva $\mathrm{IgG}$ positivity and is expected from mathematical models for leishmaniasis transmission (Chaves and Hernandez, 2004; Chaves et al. 2007; Reed et al. 2016). Moreover, studies in endemic VL areas also suggest that natural exposure to uninfected phlebotomine bites could influence the epidemiology of the disease. The convex relationship between exposure and parasitic prevalence is similar to what has been observed in other vector-borne diseases like malaria where exposure and parasitic loads have a similar correlation pattern (Ofulla et al. 2005; Chaves et al. 2009).

Vinhas et al. (2007) suggested that Lu. longipalpis exposure elicited a high sand fly saliva antibody response in humans exposed to bites over long periods of time, and that re-exposure caused significant increase of antibody levels; as it was also demonstrated by other authors that anti-saliva immune responses are dose-dependent (Hostomska et al. 2008). However, Leishmania spp. infections in mice that got repeatedly exposed to infective bites and that had high levels of anti-saliva antibodies, showed some protection against Leishmania spp. infection following short-term exposure to infective bites (Rohoušová et al. 2011). It is also worth mentioning that Valenzuela et al. (2001) showed that sand fly salivary proteins were able to protect vaccinated mice challenged with parasites plus saliva, because the vaccine produced both intense humoral and delayed-type hypersensitivity (DTH) reactions. However, B cell-deficient mice immunized with the plasmid vaccine successfully controlled Leishmania infection when injected with parasite plus saliva. These results indicate that DTH response against saliva provides most or all of the protective effects of this vaccine and that salivary gland proteins or their cDNAs are viable vaccine targets against leishmaniasis (Valenzuela et al. 2001). This result is strong evidence that saliva-specific $\mathrm{T}$ cells producing IFN- $\gamma$, in the form of a DTH reaction at the bite site, is likely the major protective response against Leishmania spp. parasites (Gomes and Oliveira, 2012).

The increased odds of infection and parasitic loads in males over females could reflect differential patterns of exposure, like observed in the epidemiology of the leishmaniasis in the New World (Christensen et al. 1983; Matlashewski et al. 2011; Alvar et al. 2012), but further epidemiological studies assessing the occupation of individuals are necessary to better understand whether gender differences are related to differential parasite exposure.

The multiple correspondence analysis considering the clinical-immunological profile and diagnostic tests for $L$. (L.) $i$. chagasi infection showed a lack of association between clinical signs, sex, seropositivity for anti-saliva antibodies. This result suggests that clinical symptoms of VL are unlikely to be 
associated with sand fly saliva exposure. In contrast, individuals who tested positive in the LST tests were likely to show AI, while individuals who test positive for IFAT were associated with III and SOI. However, individuals with SRI were likely to be positive by either LST or IFAT test. Thus, classical diagnostic test like as LST and IFAT are associated with specific clinical VL manifestations, highlighting the usefulness of these diagnostics for clinical management of VL patients (Crescente et al. 2009; Silveira et al. 2010).

Finally, our results suggest that increased exposure to sand fly saliva and/or parasites could be associated with anti-Leishmania protective immune mechanisms. One possible way to better understand the role of sand fly bite exposure would be by measuring domiciliary and peridomiciliary sand fly abundance, since sand fly abundance has been associated with leishmaniasis prevalence not only at the household level (Saldaña et al. 2013), but also at regional geographical scales (Anderson et al. 2011; Chaves et al. 2014; Yamada et al. 2016).

\section{ACKNOWLEDGEMENTS}

L.F.C. is thankful to Ms. Junko Sakemoto for valuable administrative support at Nagasaki University and Maria da Gloria Teixeira Sousa for critical review.

\section{FINANCIAL SUPPORT}

São Paulo Research Foundation-Hebrew University of Jerusalem (FAPESP-HUJ) collaborative grant \#2011/ 51404-9, Brazilian Ministry of Health and LIM50 HCFMUSP. M.D.L. is a Research Fellows from CNPq, Brazil. L.F.C. was funded by Nagasaki University (Program for Nurturing Global Leaders in Tropical and Emerging Communicable Diseases).

\section{REFERENCES}

Abbasi, I., Aramin, S., Hailu, A., Shiferaw, W., Kassahun, A., Belay, S., Jaffe, C. and Warburg, A. (2013). Evaluation of PCR procedures for detecting and quantifying Leishmania donovani DNA in large numbers of dried human blood samples from a visceral leishmaniasis focus in Northern Ethiopia. BMC Infectious Diseases 13, 153.

Alvar, J., Vélez, I. D., Bern, C., Herrero, M., Desjeux, P., Cano, J., Jannin, J., Boer, M. D. and the, W. H. O. L. C. T. (2012). Leishmaniasis worldwide and global estimates of its incidence. PLoS ONE 7, e35671.

Amora, S. S., Bevilaqua, C. M., Dias Ede, C., Feijo, F. M., Oliveira, P. G., Peixoto, G. C., Alves, N. D., Oliveira, L. M. and Macedo, I. T. (2010). Monitoring of Lutzomyia longipalpis Lutz \& Neiva, 1912 in an area of intense transmission of visceral leishmaniasis in Rio Grande do Norte, Northeast Brazil. Revista Brasileira de Parasitologia Veterinaria 19, 39-43.

Anderson, R. M. and May, R. M. (1991). Infectious Diseases of Humans: Dynamics and Control. Oxford University Press, Oxford.

Anderson, J. M., Samake, S., Jaramillo-Gutierrez, G., Sissoko, I., Coulibaly, C. A., Traoré, B., Soucko, C., Guindo, B., Diarra, D., Fay, M. P., Lawyer, P. G., Doumbia, S., Valenzuela, J. G. and Kamhawi, S. (2011). Seasonality and prevalence of Leishmania major infection in Phlebotomus duboscqi Neveu-Lemaire from two neighboring villages in Central Mali. PLoS Neglected Tropical Diseases 5, e1139.

Barral, A., Honda, E., Caldas, A., Costa, J., Vinhas, V. and Rowton, E. D. (2000). Human immune response to sand fly salivary gland antigens: a useful epidemiological markers? American Fournal of Tropical Medicine and Hygiene 62, 740-745.
Bolker, B. M., Brooks, M. E., Clark, C. J., Geange, S. W., Poulsen, J. R., Stevens, M. H. H. and White, J.-S. S. (2009). Generalized linear mixed models: a practical guide for ecology and evolution. Trends in Ecology \& Evolution 24, 127-135

Calzada, J. E., Saldaña, A., González, K., Rigg, C., Pineda, V., Santamaría, A. M., Rodriguez, I., Gottdenker, N., Laurenti, M. D. and Chaves, L. F. (2015). Cutaneous Leishmaniasis in dogs: is high seroprevalence indicative of a reservoir role? Parasitology 142, 1202-1214.

Chaves, L. F. (2010). An entomologist guide to demystify pseudoreplication: data analysis of field studies with design constraints. Fournal of Medical Entomology 47, 291-298.

Chaves, L. F. and Hernandez, M.-J. (2004). Mathematical modelling of American Cutaneous Leishmaniasis: incidental hosts and threshold conditions for infection persistence. Acta Tropica 92, 245-252.

Chaves, L. F., Hernandez, M.-J., Dobson, A. P. and Pascual, M. (2007). Sources and sinks: revisiting the criteria for identifying reservoirs for American cutaneous leishmaniasis. Trends in Parasitology 23, 311-316.

Chaves, L. F., Kaneko, A. and Pascual, M. (2009). Random, top-down, or bottom-up coexistence of parasites: malaria population dynamics in multi-parasitic settings. Ecology 90, 2414-2425.

Chaves, L. F., Morrison, A. C., Kitron, U. D. and Scott, T. W. (2012). Nonlinear impacts of climatic variability on the density-dependent regulation of an insect vector of disease. Global Change Biology 18, 457-468.

Chaves, L. F., Calzada, J. E., Valderama, A. and Saldaña, A. (2014). Cutaneous Leishmaniasis and Sand Fly fluctuations are associated with El Niño in Panamá. PLoS Neglected Tropical Diseases 8, e3210.

Christensen, H. A., Fairchild, G. B., Herrer, A., Johnson, C. M., Young, D. G. and Vasquez, A. M. D. (1983). The ecology of cutaneous leishmaniasis in the republic of Panama. Fournal of Medical Entomology 20, 463-484.

Confalonieri, U. E. C., Margonari, C. and Quintão, A. F. (2014) Environmental change and the dynamics of parasitic diseases in the Amazon. Acta Tropica 129, 33-41.

Costa, C. H. (2008). Characterization and speculations on the urbanization of visceral leishmaniasis in Brazil. Cadernos de saude publica 24, 2959-2963. Crescente, J. A., Silveira, F. T., Lainson, R., Gomes, C. M., Laurenti, M. D. and Corbett, C. E. (2009). A cross-sectional study on the clinical and immunological spectrum of human Leishmania (L.) infantum chagasi infection in the Brazilian Amazon region. Transactions of the Royal Society of Tropical Medicine and Hygiene 103, 1250-1256.

Desjeux, P. (2001). Worldwide increasing risk factors for leishmaniasis. Medical Microbiology and Immunology 190, 77-79.

Desjeux, P. (2004). Leishmaniasis: current situation and new perspectives. Comparative Immunology Microbiology and Infectious Diseases 27, 305-318. Faraway, J. J. (2004). Linear Models with R. CRC Press, Boca Raton. Finch, W. H., Bolin, J. E. and Kelley, K. (2014). Multilevel Modeling Using R. CRC Press, Boca Rat'on, FL, USA.

Garcia, L., Kindt, A., Bermudez, H., Llanos-Cuentas, A., De Doncker, S., Arevalo, J., Quispe Tintaya, K. W. and Dujardin, J.-C. (2004). Culture-independent species typing of neotropical Leishmania for clinical validation of a PCR-based assay targeting heat shock protein 70 genes. Fournal of Clinical Microbiology 42, 2294-2297.

Gomes, R. and Oliveira, F. (2012). The immune response to sand fly salivary proteins and its influence on Leishmania immunity. Frontiers in Immunology 3, 110.

Gomes, R. B., Brodskyn, C., de Oliveira, C. I., Costa, J. and Miranda, J. C. (2002). Seroconversion against Lutzomuia longipalpis saliva concurrent with the development of anti-Leishmania chagasi delayed-type hyprsensitivity. Fournal of Infectious Diseases 186, 1530-1534. Harhay, M. O., Olliaro, P. L., Costa, D. L. and Costa, C. H. (2011). Urban parasitology: visceral leishmaniasis in Brazil. Trends in Parasitology 27, 403-409.

Hostomska, J., Rohousova, I., Volfova, V., Stanneck, D., Mencke, N. and Volf, P. (2008). Kinetics of canine antibody response to saliva of the sand fly Lutzomyia longipalpis. Vector Borne and Zoonotic Diseases 8, $443-450$.

Kamhawi, S., Belkaid, Y., Modi, G., Rowton, E. and Sacks, D. (2000). Protection against cutaneous leishmaniasis resulting from bites of uninfected sand flies. Science 290, 1351-1354.

Kaneko, A., Taleo, G., Kalkoa, M., Yaviong, J., Reeve, P. A., Ganczakowski, M., Shirakawa, C., Palmer, K., Kobayakawa, T. and Bjorkman, A. (1998). Malaria epidemiology, glucose 6-phosphate dehydrogenase deficiency and human settlement in the Vanuatu Archipelago. Acta Tropica 70, 285-302.

Kaneko, A., Chaves, L. F., Taleo, G., Kalkoa, M., Isozumi, R., Wickremasinghe, R., Perlmann, H., Takeo, S., Tsuboi, T. and Tachibana, S.-I. (2014). Characteristic age distribution of plasmodium 
vivax infections after malaria elimination on aneityum Island, Vanuatu. Infection and Immunity 82, 243-252.

Lainson, R. and Rangel, E. F. (2005). Lutzomyia longipalpis and the ecoepidemiology of American visceral leishmaniasis, with particular reference to Brazil: a review. Memorias do Instituto Oswaldo Cruz 100, 811-827.

Lainson, R. and Shaw, J. (2005). Leishmaniasis in the New World. In Topley $\sigma^{\circ}$ Wilson's Microbiology and Microbial Infections, Vol. 5, Parasitology (ed. Collier, L., Sussman, A. B. M.), pp. 313-349. Arnold, London.

Lima, L. V. R., de Souza, A. A. A., Jennings, Y. L., Corrêa, Z., de Jesus, R., Everdosa, D., Ayres, M. and Silveira, F. T. (2003). Comparison of the reactivity between antigens of Leishmania (L.) chagasi, L. (L.) amazonensis e Leishmania sp. (Bio-Manguinhos) in the sero-diagnosis of visceral leishmaniasis by the indirect fluorescent antibody test (IFAT). Revista do Instituto de Medicina Tropical de Sao Paulo 45 s147.

Maia-Elkhoury, A. N., Alves, W. A., Sousa-Gomes, M. L., Sena, J. M. and Luna, E. A. (2008). Visceral leishmaniasis in Brazil: trends and challenges. Cadernos de saude publica 24, 2941-2947.

Matlashewski, G., Arana, B., Kroeger, A., Battacharya, S., Sundar, S., Das, P., Sinha, P. K., Rijal, S., Mondal, D. Zilberstein, D. and Alvar, J. (2011). Visceral leishmaniasis: elimination with existing interventions. The Lancet Infectious Diseases 11, 322-325.

Michel, G., Pomares, C., Ferrua, B. and Marty, P. (2011). Importance of worldwide asymptomatic carriers of Leishmania infantum (L. chagasi) in human. Acta Tropica 119, 69-75.

Nicolas, L., Milon, G. and Prina, E. (2002). Rapid differentiation of Old World Leishmania species by LightCycler polymerase chain reaction and melting curve analysis. Fournal of Microbiological Methods 51, 295-299.

Ofulla, A. V., Moormann, A. M., Embury, P. E., Kazura, J. W. Sumba, P. O. and John, C. C. (2005). Age-related differences in the detection of Plasmodium falciparum infection by PCR and microscopy, in an area of Kenya with holo-endemic malaria. Annals of Tropical Medicine and Parasitology 99, 431-435.

Prothero, R. M. (1965). Migrants and Malaria. Longmans, London, UK. Reed, S. G., Coler, R. N., Mondal, D., Kamhawi, S. and Valenzuela, J. G. (2016). Leishmania vaccine development: exploiting the host-vector-parasite interface. Expert Review of Vaccines 15, 81-90.

Rohousova, I., Ozensoy, S., Ozbel, Y. and Volf, P. (2005). Detection of species-specific antibody response of humans and mice bitten by sand flies. Parasitology 130, 493-499.

Rohoušová, I., Hostomská, J., Vlková, M., Kobets, T., Lipoldová, M. and Volf, P. (2011). The protective effect against Leishmania infection conferred by sand fly bites is limited to short-term exposure. International Fournal for Parasitology 41, 481-485.

Saldaña, A., Chaves, L. F., Rigg, C. A., Wald, C., Smucker, J. E. and Calzada, J. E. (2013). Clinical cutaneous leishmaniasis rates are associated with household Lutzomyia gomezi, Lu. panamensis, and Lu. trapidoi abundance in trinidad de Las Minas, Western Panama. The American Fournal of Tropical Medicine and Hygiene 88, 572-574.

Salomón, O., Feliciangeli, M. D., Quintana, M., Afonso, M. D. S. and Range1, E. (2015). Lutzomyia longipalpis urbanisation and control. Memorias do Instituto Oswaldo Cruz 110, 831-846.

Saraiva, L., Andrade Filho, J. D., Falcao, A. L., de Carvalho, D. A., de Souza, C. M., Freitas, C. R., Gomes Lopes, C. R., Moreno, E. C. and Melo, M. N. (2011). Phlebotominae fauna (Diptera: Psychodidae) in an urban district of Belo Horizonte, Brazil, endemic for visceral leishmaniasis: characterization of favored locations as determined by spatial analysis. Acta Tropica 117, 137-145.

Silveira, F. T., Lainson, R., Pereira, E. A., de Souza, A. A., Campos, M. B., Chagas, E. J., Gomes, C. M., Laurenti, M. D. and Corbett, C. E. (2009). A longitudinal study on the transmission dynamics of human Leishmania (Leishmania) infantum chagasi infection in Amazonian Brazil, with special reference to its prevalence and incidence. Parasitology Research 104, 559-567.

Silveira, F. T., Lainson, R., De Souza, A. A., Campos, M. B., Carneiro, L. A., Lima, L. V., Ramos, P. K., de Castro Gomes, C. M. Laurenti, M. D. and Corbett, C. E. (2010). Further evidences on a new diagnostic approach for monitoring human Leishmania (L.) infantum chagasi infection in Amazonian Brazil. Parasitology Research 106, 377-386.

Solano-Gallego, L., Miró, G., Koutinas, A., Cardoso, L., Pennisi, M G., Ferrer, L., Bourdeau, P., Oliva, G. and Baneth, G. (2011). LeishVet guidelines for the practical management of canine leishmaniosis. Parasites E Vectors 4, 1

Valenzuela, J. G., Belkaid, Y., Garfield, M. K., Mendez, S., Kamhawi, S., Rowton, E. D., Sacks, D. L. and Ribeiro, J. M. (2001). Toward a defined anti-Leishmania vaccine targeting vector antigens: characterization of a protective salivary protein. Fournal of Experimental Medicine 194, 331-342.

Venables, W. N. and Ripley, B. D. (2002). Modern Applied Statistics with $S$. Springer, New York.

Vinhas, V., Andrade, B. B., Paes, F., Bomura, A., Clarencio, J., Miranda, J. C., Báfica, A., Barral, A. and Barral-Netto, M. (2007) Human anti-saliva immune response following experimental exposure to the visceral leishmaniasis vector, Lutzomyia longipalpis. European fournal of Immunology 37, 3111-3121.

Volf, P. and Rohoušová, I. (2001). Species-specific antigens in salivary glands of phlebotomine sandflies. Parasitology 122, 37-41.

Wijeyaratne, P. M., Arsenault, L. K. J. and Murphy, C. J. (1994) Endemic disease and development: the leishmaniases. Acta Tropica $\mathbf{5 6}$ 349-364. doi: http://dx.doi.org/10.1016/0001-706X(94)90106-6

Yamada, K., Valderrama, A., Gottdenker, N., Cerezo, L., Minakawa, N., Saldaña, A., Calzada, J. E. and Chaves, L. F. (2016) Macroecological patterns of American Cutaneous Leishmaniasis transmission across the health areas of Panamá (1980-2012). Parasite Epidemiology and Control 1, 42-55. 